[16] E. Pozzi-Escot. Le dosage rapide de la matière grasse du lait. Gerber ou Babcock ? Le Lait, 1926, 6, 601.

[17] F. Richard. Analyse de la poudre de lait. Le Lait, 1926, 6, 86.

[18] G. Roender. Gerber ou Babcock ? Le Lait, 1927, 7, 354.

[19] Röse-Gottlieb (Méthode de). Les méthodes officielles américaines d'analyse du lait. Le Lait, 1926, 6, 54.

[20] A. Schoonjans. Note sur le dosage de la matière grasse dans les poudres de lait. Le Lait, 1925, 5, 782.

[21] M. Siegreld, Untersuchungen über die Zusammensetzung des Butterfettes. Zeitschr. f. Untersuch. d. Nahrungs-u. Genussmittel, 1912, 24, 453.

[22] A. Villiters, E. Colitn et M. Fayolie. Aliments lactés et aliments gras, Paris, Doin, éd., 1911.

[23] R. Vladesco. La solubilisation de la matière organique du lait. Ses applications. Le Lrit, 1925, 5, 479.

\title{
VARIATIONS SAISONNIÈRES ET CYCLE ANNUEL DES INDICES DES BEURRES DU NORD DE LA FRANCE
}

\author{
par
}

le Docteur Michel POLONOVSKI et Mile Marcelle THOMAS, Professeur de Chimie Biologique

Docteur en Pharmacie Assistante de Chimie Biologique

à la Faculté de Médecine et de Pharmacie de Lille.

\section{(Suite).}

\section{DISCUSSION DES RÉSULTATS.}

De toute cette analyse descriptive, il ressort que chaque série de beurres présente des variations individuelles, mais que dans toutes ces irrégularités, il va être possible de trouver une régularité.

Schématisons : nous aboutissons à une courbe passant par un minimum situé entre deux maxima. Le minimum correspond à la bonne saison ; les deux maxima à la mauvaise saison.

Si le début de ce travail avait coïncidé avec le début d'une saison, les trois stades que la courbe comportent en apparence n'auraient plus été que deux : le stade d'hiver, le stade d'été.

1. VARIATIONS SAISONNIËRES. - Les réactions sont si brusques qu'aucun doule n'est possible. Du 8 au 17 Qctobre, les beurres de Phalempin ont passé d'un indice de saponification de 225 à un indice de 239, l'indice d'iode tombant dans le même temps de 32 à 23.

A. Winnezeele, le beurre du 22 Octobre donne un indice de saponification de 214,2 , celui du 4 Novembre a monté à 222 , celui du 18 Novembre est à 237. L'indice d'iode descend corrélativement de 47,6 à 24,7 .

Dans tous les graphiques, même phénomène typique. A ce changement brutal fait suite une longue période à caractères 
durables et constants ; il n'y a pas confusion possible de valeurs pour les indices d'hiver et d'été d'une série de beurres. Ils oscillent dans des plans parfaitement séparés.

Donc, suivant que les vaches vivent à l'étable ou en pâture, le beurre est de composition différente.

En étable, elles sont immobiles, dans une atmosphère tiède, uniforme, à l'abri des variations atmosphériques. Elles reçoivent une provende substantielle, rationnellement mesurée.

Dès les premiers beaux jours, on les habitue à la pâture, et au bout d'une semaine ou deux, la plupart des fermiers les y laissent nuit et jour durant la belle saison. Elles vont y connaître tout l'imprévu de la vie au grand air. Elles vont brouter à loisir les herbes dont elles sont friandes.

Deux conditions importantes ont varié : le milieu, l'alimentation. Ont-elles toutes deux même influence, en ce qui concerne les variations saisonnières ?

Une circonstance particulièrement heureuse va nous permettre d'éliminer une variable : Quelle que soit la saison, les vaches de Phalempin rentrent tous les soirs à l'étable. Elles ont été nourries d'herbe à partir du $1^{\text {er }}$ Mai : c'est à partir du 1er Mai que l'affaissement des indices a lieu, et quel que soit le temps, nous l'avons vu, nous ne retrouverons pas les hauts indices de l'hiver.

Ils réapparaissent avec la nourriture artificielle, le 17 Octobre. Or, fait très important, les vaches ont continué à vivre tout le jour en pâture et n'ont repris que le 20 Novembre la stabulation totale.

Est-ce à dire que le temps a été favorable ? Pas du tout. La température moyenne de $10^{\circ} 7$ en Avril n'est plus que de $8^{\circ} 6$ en Novembre. Les averses sont fréquentes et violentes. Pluie, vent et même grêle font rage. Le 2 et le 3 Novembre, la tempête fut telle que le baromètre descendit à $731 \mathrm{~m} / \mathrm{m} 2$. Les vaches sont en pâture quand même, car l'accalmie du matin fait espérer - à tort - le retour d'un temps plus doux; la tempête ne s'élève dans toute sa violence que vers le milieu de la journée.

Quels vont être les indices des beurres de cette époque?

$\begin{array}{ccc}\text { Ind. Koettströfer } & \text { Ind. Planchon } & \text { Ind. Leffman-Beam } \\ 238,5 & 28,7 & 27,5 \\ 237,2 & 26,6 & 27\end{array}$

Les indices de Ketrstörfer et les indices de Planchon en particulier ne sont jamais montés si haut.

A l'opposé de ces indices, nous pouvons citer ceux des trois beurres du Pas-de-Calais dont nous avons parlé page 254; ils sont anormalement bas : à peine égaux à 208 (indice de saponification) 
et à 16 (indice d'A. V. S.), et pourtant il s'agit de vaches soumises d'un bout de l'année à l'autre à la tiède ambiance de l'étable.

Donc, sans aucun conteste, les variations saisonnières des indices des beurres sont les conséquences des variations saisonnières de la nourriture.

2. VARiations plurihebdomadaires. - Elles n'ont pas la belle ordonnance régulière des précédentes. Elles n'en ont pas les débuts franes, ni les caractères constants. De forme, de durée variables, elles ne sont pas négligeables cependant, car tous les beurres présentent aux mêmes moments, cette même succession d'étapes.

A. Saison d'été. - Trois périodes : $1^{\circ}$ En Mai et Juin une descente progressive pour les séries de Steenvoorde, de Winnezeele (il ne s'agit jamais des indices d'iode quand ils ne sont pas mentionnés expressément), un plateau d'indices intermédiaires entre les indices d'hiver et les indices des mois suivants pour Vendegies, un plateau bas pour les acides solubles et volatils solubles de Phalempin.

Nous sommes dans la période chaude du printemps, l'herbe gorgée de sève est fine, savoureuse, et riche de tous les principes qu'elle élabore pour la fleur et le fruit ensuite. Elle n'a pas encore été pâturée.

Partout les indices se maintiennent à des taux satisfaisants. A Vendegies les indices de saponification sont au niveau des indices d'hiver; cette particularité est probablement due à l'étendue des pâturages. A peine l'herbe diminue-t-elle dans un pré, que les vaches la retrouvent en abondance dans un autre. Elles peuvent manger sans se déplacer, ce qui est un grand principe de zootechnie.

En tout cas, quels que soient les beurres examinés, les indices de KotTstörfer ne sont pas au-dessous de 220 ; les indices d'A. V. S., à peine au-dessous de 22.

20 Une secondé période de Juillet à Octobre. - L'affaissement est général, sauf pour Phalempin; ce qui n'a rien de surprenant, car ici encore la divergence des conditions d'alimentation va nous être précieuse. Le mois de Juillet en premier lieu, est pour tous les beurres une période de baisse qui ne comporte pas d'exception. Partout, les courbes prennent une forme d'entonnoir. Le temps est en effet particulièrement inclément : l'orage gronde tous les jours accompagné d'éclairs et de grêle pendant toute la première semaine. Ensuite, l'atmosphère reste lourde et orageuse, sans que pour cela il cesse de pleuvoir : souvenons-nous de l'énorme quantité d'eau tombée pendant tout le mois : $127 \mathrm{~m} / \mathrm{m} 3$, le maximum de toutes les hauteurs des pluies tombées en Juilllet pendant 9 ans.

Dans les pâtures, les vaches apeurées mugissent lamentable- 
ment. Elles se cherchent, inquiètes, tandis que de sinistres nuages noirs roulent très bas, impatients de crever en averses tourbillonnantes. Comment brouter et ruminer paisiblement sous le fouet de telles rafales?

En Août survient l'accalmie, mais déjà la nature a perdu toute force vive. Le soleil s'attarde sous d'autres cieux. L'herbe pâturée repousse sans entrain ; celle qui ne l'a pas été n'est plus que le soutien tôt flétri de ses graines.

C'est le moment où les indices de saponification de Steenvoorde avoisinent 220, et ceux de Winnezeele 215. A Vendegies, la chute se poursuit, présentant à peine une ou deux ébauches de rémittence, de 226 à 215 .

A Steenvoorde les A. V. S. sont entre 21 et 23 , à Winnezeele entre 15 et 24. A Vendegies, la chute ne s'arrête pas avant 18,8 .

Rien de tel à Phalempin. Les vaches y ont, comme dans les autres fermes, souffert du mauvais temps de Juillet et leur beurre s'en est ressenti, mais dès le début d'Août, il leur a été octroyé tous les jours en supplément de l'herbe broutée et des tourteaux d'arachides, de la luzerne et de l'herbe anglaise coupées. (Voir tableau de l'alimentation, page 252).

Donc, lorsque l'herbe manque, que la vache soumise à un régime de famine réduite est dans un état de misère physiologique, il y a répercussion très nette sur le beurre et baisse prononcée des indices.

Cet affaissement n'a pas lieu lorsque les fermiers, loin d'abandonner à la nature le soin exclusif de nourrir leurs bêtes, se préoccupent de remédier aux carences de l'affouragement naturel par un affouragement artificiel rationnellement dispensé.

$3^{\mathrm{e}}$ période : Octobre. - La moyenne des températures nocturnes a baissé de $3^{\circ}\left(11^{\circ}\right.$ à $\left.8^{\circ}\right)$. Les nuits sont trop fraîches pour laisser les vaches les passer au pré.

A l'étable, les fermiers ont toute facilité pour donner au troupeau une ration complémentaire de feuilles de betteraves qu'on vient de rentrer. Partout où ils s'en préoceupent, les indices remontent:

A Steenvoorde, les indices de saponification atteignent 225 ; les indices d'acides volatils, 27. A Troisvilles, il en est de même : les vaches ont été menées dans des pâtures quittées depuis longtemps, l'herbe a eu le temps de s'y renouveler et lorsqu'elle a manqué on l'a suppléée par des feuilles de betteraves.

B. Saison d'hiver, - Elle ne coïncide pas avec le moment où les vaches restent définitivement à l'étable, mais avec la période où le travail des betteraves étant plus avaneé, les fermiers ont à leur disposition des collets de betteraves à sucre.

Unissons par la pensée les deux extrémités de chacune de nos courbes. L'opération est logique. Nos résultats sont le fruit d'une 
année complète d'observations; les conditions de vie des vaches ne varient pas d'une année à l'autre, et la preuve la plus irréfutable de ces raisons est dans le fait que les courbes se rejoignent au même niveau.

Le cycle étant fermé de la sorte, nous avons une vue d'ensemble de la saison d'hiver, autrement dit la saison de la nourriture artificielle exclusive.

Elle n'a pas les étapes marquées de la saison d'été. Les vaches vont connaître à l'étable une vie végétative d'où tout imprévu est exclu : température égale, aux mêmes heures les mêmes aliments. Et pourtant, dès que survient un changement un peu notable dans la composition de ceux-ci, le beurre s'en ressent, et imprime à la courbe un mouvement d'ascension ou de chute.

Sur ces plateaux élevés que représentent les indices d'hiver, deux surélévations : en fin d'Octobre, et en Novembre, époque des collets de betteràves à sucre; c'est la période de gloire des indices.

A Winnezeele :

$\begin{array}{cccc}\begin{array}{c}\text { Ind. } \\ \text { Koettstörfer }\end{array} & \begin{array}{c}\text { Ind. } \\ \text { Planchon }\end{array} & \begin{array}{c}\text { Ind. } \\ \text { Leffman-Beam }\end{array} & \begin{array}{c}\text { Ind. } \\ \text { Polenske }\end{array} \\ 237 & 28,2 & 27,6 & 4,1 \\ 234,5 & 27,4 & 32,3 & 4,7 \\ 236,4 & 25,3 & 31,1 & 5,1\end{array}$

A Phalempin, dès le 17 Octobre les indices de saponification avoisinent 240 , les indices d'acides solubles 28 , les indices d'acides volatils solubles 27 et les indices d'acides volatils insolubles 4 et 5 . (Nous avions débuté par ces hauts indices avec un beurre analysé le 26 Novembre 1929.)

A Troisvilles, même richesse d'indices.

A Steenvoorde, les beurres de laiterie plus modérés gardent une place honorable.

A Vendegies, les betteraves à sucre ne constituent pas le fond de la nourriture des vaches, l'approvisionnement en est peu facile ; les indices se relèvent à la façon des collets de betteraves fourragères, mais beaucoup moins magnifiquement.

Au Ier Décembre, il n'y a plus un collet de betteraves à sucre dans toute la région du Nord. Les vaches vont trouver dans leur mangeoire tourteaux et betteraves fourragères avec du son et des paillettes de blé pour leur combler l'estomac.

Décembre et Janvier pourraient constituer la deuxième période de l'hiver.

La troisième période débuterait dans le courant de Février, 
jusqu'en Mars. Elle est inégalement marquée par les différentes courbes.

A Steenvoorde et à Winnezeele, les indices de saponification et d'acides volatils solubles montent.

A Phalempin, ee sont surtout les A. V. S.

A Vendegies, ee sont les mêmes qu'à Steenvoorde et à Winnezeele, accompagnés des acides solubles.

La cause pourrait en être due aux pulpes de betteraves à sucre que les distilleries et les raffineries livrent à cette époque. Ce n'est là qu'une suggestion, mais assez vraisemblable, si l'on songe que, de longues et minutieuses enquêtes, il résulte que e'est la seule modification signalée dans la vie des vaches à ce moment.

Les fermiers augmentent journellement la proportion de pulpes, et ceci expliquerait la montée progressive des courbes.

Nous sommes revenus au fléchissement des indices d'été. Le cycle est terminé.

3. VARIATIONS INdividuelles. - L'examen le plus superficiel des graphiques ne peut les méconnaître : le graphique de Steenvoorde même n'est pas beaucoup moins hérissé que les autres.

Toutefois les oscillations brusques sont rares. Elles apparaissent aux changements de régime : par exemple, les vaches sont en pâture pendant une période de quelques beaux jours; qu'une tempête survienne, les voilà pour plusieurs jours ramenées à l'étable, au régime d'hiver.

C'est ainsi qu'à Phalempin, vers le 7 ou le 8 Avril, le temps est sec, la température clémente, le baromètre très haut; on mène les vaches en pâture, d'où chute des indices très brusques pour l'indice de KGTtstöRfer : 234,2 à 226,1, notable pour l'indice de Leffmann-Beam : 24 à 22,4 . Mais le beau temps ne dure pas, les vaches retrouvent à l'étable leurs repas de pulpes et de tourteaux, et le beurre ses indices élevés : 232,7-25,6, jusqu'à l'avènement définitif de la saison d'été.

Oscillation brusque aussi quand le fermier pour une raison de commodité diminue la ration de collets de betteraves à sucre, ainsi que le témoignent les beurres de Phalempin du 17 Octobre au 5 Novembre :

Ind. Kottstorfer

\begin{tabular}{|c|c|}
\hline 17 octobre & $\ldots \ldots \ldots$ \\
\hline 23 octobre & $\ldots$ \\
\hline 30 octobre & $\ldots$ \\
\hline 5 novembr & \\
\hline
\end{tabular}

Ind. Leff man Beam

$\begin{array}{ll}29,7 & 32,5 \\ 26,8 & 24,3 \\ 29,5 & 29,1 \\ 30 & 28,7\end{array}$


et les beurres de Phalempin du 14 au 27 Novembre :

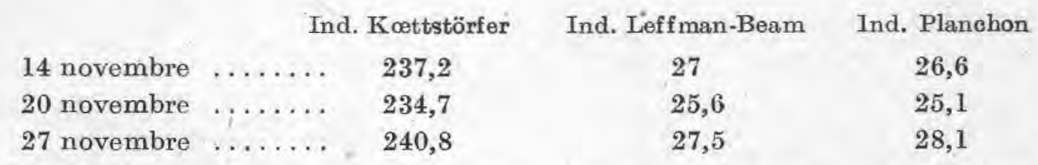

La chute rapide se borne aux indices d'acides solubles et d'acides volatils solubles lorsqu'à Phalempin, le 25 Février et le 5 Mars, les vaches reçoivent des pulpes de distillerie à peu près dépourvues de tout principe sucré.

$\begin{array}{cc}\text { Ind. Leffman-Beam } & \text { Ind. Planchon } \\ 32,1 & 24,8 \\ 24,6 & 24,7 \\ 25 & 22,9 \\ 29,8 & 24,3\end{array}$

Signalons encore le brusque début des courbes de Phalempin : elles descendent une pente raide du 28 Novembre au 2 Décembre. Nous ne nous y attarderons pas, car il ne s'agit pas ici d'une oscillation, mais du commencement de la deuxième période de la saison d'hiver dont nous avons longuement parlé dans le paragaphe précédent.

Il est encore dẹs écarts rapides et considérables pour lesquels une explication certaine nous manque.

Pourquoi le 4 Décembre 1929, à Steenvoorde, l'indice de PlanCHON s'élance-t-il de 26,6 à 37 pour retomber à 28,7 , et les 11 et 12 Décembre se dresse-t-il à 33 et 32,5 entre deux indices de 26,5 et de 26,1 ?

Il est à remarquer que Vendegies, le seul de tous les lieux d'origine des beurres analysés qui ne soit pas un pays où règne la betterave à sucre, ne présente pas ces écarts désordonnés.

Ils ne sont d'ailleurs pas toujours ni aussi brusques ni aussi déconcertants. Dépressions et élévations, influencées par le temps, mettant le plus souvent plusieurs semaines à progresser et à déeliner. On les reconnaît au mouvement d'ensemble qu'elles impriment aux courbes; la composition des beurres se révèle en effet comme un baromètre très sensible et très juste. Et pendant tout l'été, il y a une relation étroite entre la météorologie et l'allure des courbes.

C'est une première dépression générale causée par le temps orageux de la fin de Mai : tous les jours le ciel est zébré d'éclairs.

Les indices se redressent pour retomber dans la grande dépression de Juillet sur laquelle nous avons insisté précédemment. La saturation du sol en eau est telle que les cours d'eau débordent partout.

C'est enfin le brouillard intense des derniers jours de Septembre et du début d'Oetobre (visibilité maximum : $50 \mathrm{~m} / \mathrm{m}$ à 7 heures). Il 
stagne alors sur les prés des Flandres et la matinée est avancée quand il se dissipe. L'après-midi, il retombe en pluie. En conséquence, les indices se dépriment fortement (1):
A Winnezeele $\left\{\begin{array}{r}30 \text { sept. } \\ 8 \text { oct. } \\ 14 \text { oct. }\end{array}\right.$
A. V. S. $\left\{\begin{array}{l}24 \\ 15 \\ 23,2\end{array}\right.$
Ac. sol. $\left\{\begin{array}{l}25,9 \\ 20,2 \\ 28,9\end{array}\right.$

Le 22 Octobre, même phénomène, dû à la même cause :

$$
\text { Soit }\left\{\begin{array} { r } 
{ 1 4 \text { oct. } } \\
{ 2 2 \text { oct. } } \\
{ 4 \text { nov. } }
\end{array} \text { A. V. S. } \left\{\begin{array} { l } 
{ 2 3 , 2 } \\
{ 1 6 , 8 } \\
{ 2 5 , 3 }
\end{array} \quad \text { Ac. sol. } \left\{\begin{array}{l}
28,9 \\
21,5 \\
25,8
\end{array}\right.\right.\right.
$$

Avant de clore ce chapitre sur les variations des beurres et leurs causes, signalons en passant la curieuse hausse des beurres de Troisvilles du 12 au 20 Septembre :

$$
12 \text { sept: }\left\{\text { sept. } \left\{\text { Ind.desaponification } \left\{\begin{array} { l } 
{ 2 1 6 , 8 } \\
{ 2 1 8 , 4 }
\end{array} \text { A. V. S. } \left\{\begin{array} { l } 
{ 2 1 , 9 } \\
{ 2 4 , 2 }
\end{array} \text { Ac. sol. } \left\{\begin{array}{l}
22,8 \\
23,8
\end{array}\right.\right.\right.\right.\right.
$$

lorsque les jeunes pousses de trèfle ont forci et fleuri.

Signalons en dernier lieu à la laiterie de Steenvoorde entre le 15 et le 22 Décembre 1929, l'affaisement des indices de saponification et d'acides volatils solubles (respectivement inférieurs à 230 et à 25). Il coïncide avec de graves perturbations survenues dans la fabrication du beurre à cette époque. Par suite de fermentations inexpliquées, le beurre moisissait aussitôt élaboré. Après la mise au point d'autres cultures de ferments lactiques, ces inconvénients disparurent.

De toute cette enquête sur les variations des beurres, il résulte que le rôle de l'alimentation est primordial, qu'it soit direct ou indirect.

De premier plan dans les variations saisonnières, où le seul changement de nourriture suffit à déniveler les indices. De premier plan encore dans les variations plurihebdomadaires, son influence est plus voilée dans les variations individuelles, mais apparente ou cachée, elle n'en est pas moins à peu près la seule efficace.

Les vaches ont une réputation de placidité usurpée. Elles ressentent fortement toute perturbation de leurs habitudes, et leur appétit en est diminué d'autant. Qu'elles soient impressionnées par le bien-être ou l'inquiétude, elles ne mangent plus.

Il faut les avoir vues en Avril, au sortir de l'étable, gambader dans les pâtures, s'emparant de l'espace avant de s'emparer de l'herbe embaumée, qu'elles hument de leurs naseaux grand ouverts.

Il faut les avoir vues aussi par une brûlante journée d'été,

(1) Ces écarts enlèvent beaucoup de leur valeur aux conclusions â tirer de prélèvements de comparaison, ainsi que nous l'a fait remarquer M. DoRchres, directeur du Laboratoire Municipal de Lille. 
incapables de brouter et surtout de ruminer tant les mouches les harcèlent, puis inquiètes dans l'aír qui s'alourdit, serrées les unes contre les autres et mugissant l'orage qu'elles pressentent anxieusement bien longtemps avant qu'il n'éclate.

La pluie venue, elles se serrent piteusement contre les haies, refuge bien précaire contre le réseau d'éclairs qui les enlace.

Il faut les avoir vues enfin à leur arrivée dans un parc nouveau, broyer l'herbe avidement sans prendre le temps de lever la tête et puis rêver, tandis que

$$
\begin{aligned}
& \text { "s'accomplit dans la nuit de leurs flancs } \\
& \text { "le mystère sacré qui, de ces vertes plantes } \\
& \text { "distillera pour nous les flots tièdes et blancs..." }
\end{aligned}
$$

Alors, ne pourra-t-on plus méconnaître toute l'importance de l'alimentation et de sa digestion sur la vie organique des vaches!

(A suivre.)

\section{LA MÉTHODE A.I.V. \\ POUR LA CONSERVATION DU FOURRAGE VERT}

par

le Prof. Artturi I. VIRTANEN.

Conférence faite à Stockholm, pendant la Semaine agricole

en Suède, le 15 mars 1932.

(Fin.)

Après avoir achevé les recherches sur les principes fondamentaux de la nouvelle méthode en exécutant des expériences au laboratoire, nous avons, dans l'été 1928, organisé des expériences pratiques sur une plus grande échelle. Dans ces expériences, le fourrage était en partie conservé dans des réservoirs en bois de 4.000 litres, en partie dans de grandes tours en bois de 4 mètres de diamètre ou dans des fosses en terre de 5 mètres de diamètre. L'exécution de ces expériences était absolument de nécessité, afin que la méthode fût complètement affirmée.

Ces expériences pratiques ont démontré tout d'abord que par l'arrosage du fourrage avec une solution d'acide, il était possible d'obtenir un mélange homogène. Elles ont encore affirmé qu'un hachage préalable du fourrage n'est pas nécessaire. Ces observations étaient d'une importance particulièrement grande, en ce sens que la méthode ne pouvait pas être appliquée à la pratique, si un simple arrosage par la solution d'acide se montrait insuffisant, les frais occasionnés par le hachage du fourrage étaient ainsi économisés, ce qui est un grand avantage. En résumé, ces expériences étaient 